\title{
Equilibrio ácido base \\ y espectrofotometría molecular en el espectro visible. Propuesta de enseñanza desde el enfoque Enseñanza para la Comprensión "EpC"
}

\author{
Javier A García C ${ }^{1}$ Jaga891@hotmail.com
}

\section{RESUMEN}

En este proyecto se diseñó e implementó una propuesta metodológica con estudiantes de grado once del colegio Champagnat sede Bogotá, fundamentada en el enfoque Enseñanza para la Comprensión $(\mathrm{EpC})$ para el tópico generativo de equilibrio químico ácido-base. La aplicación de la estrategia y la evaluación continua de los resultados señalan que se fomentaron los desempeños de comprensión previstos, encontrando que los estudiantes se ubican en un nivel de aprendiz según los planteamientos del enfoque educativo tomado como referencia.

Palabras clave

Enseñanza para la Comprensión, equilibrio ácido-base, espectrofotometría, desempeños de comprensión, niveles de comprensión.

\section{ABSTRACT}

In this Project was implemented a proposal methodological with students of eleventh level at Champagnat School in Bogota, making use of Teaching for Understanding (EpC) in the generative topic: equilibrium chemical acid and basic. The application of the strategic and the evaluation continuous, obtained as result the promoted the performance performances of understanding used it, finding than the students was located in apprentice level according with the structure of the educational approach than was had as reference.

Key words

Teaching for Understanding, equilibrium chemical acid and basic, spectrophotometry, performances of understanding, comprehension levels.

${ }^{1}$ Estudiante del Departamento de Química de la Universidad Pedagógica Nacional. UPN 


\section{Introducción}

La enseñanza para la comprensión es de gran importancia frente a los nuevos retos de la sociedad, pues los docentes ya no deben buscar que los estudiantes acumulen conceptos, más bien deben procurar que ellos aprendan para la vida (Martínez, 2007).

Parece ser que, aún sigue existiendo un gran abismo entre lo que los docentes aprecian y lo que los educandos comprenden (Murillo, 2006), por lo cual se asume que "el término enseñanza debe partir del conocimiento de los conceptos que manejan los estudiantes y habilidades que ellos poseen" (Stone y Wiske, 2005).

En el marco de este trabajo y desde los presupuestos teóricos del enfoque de la $\mathrm{EpC}$, se diseñan e implementan diferentes actividades para la enseñanza del equilibrio ácido base y la espectrofotometría molecular en el espectro visible. Para ello se contemplan los resultados obtenidos con el instrumento de concepciones alternativas de los estudiantes. Posteriormente, a través de la herramienta torbellino de ideas, se delimitan los desempeños que permiten caracterizar los niveles de compresión frente al tópico de estudio, de 93 estudiantes de grado once del colegio Champagnat, sede Bogotá.

La pregunta que orienta este trabajo es: ¿Cómo fomentar desempeños de comprensión en estudiantes de grado $11 \mathrm{del}$ colegio Champagnat a partir del tópico generativo del equilibrio ácido-base?

\section{Marco teórico}

\section{Enseñanza para la comprensión (EpC)}

Incluye cuatro ideas claves: Tópicos Generativos, Metas de Comprensión, Desempeños de Comprensión y Valoración Continua (Stone y Wiske, 2005).

Tópicos Generativos: Son de exploración y tienen múltiples conexiones con los intereses y experiencias son fundamentales, ya que comprometen tanto a estudiantes como a maestros y se fundan en tópicos anteriores.

Metas de Comprensión: Las afirmaciones o preguntas que expresan aquello que es más importante para los estudiantes, 0 durante un período de larga duración, como por ejemplo un año escolar (Hilos Conductores).

Desempeños de Comprensión: Son las actividades que desarrollan y demuestran la comprensión de los estudiantes acerca de la comprensión de metas, haciendo que los estudiantes utilicen lo que ya conocen.

Valoración Continua: Es el proceso por el cual los estudiantes obtienen retroalimentación continua sobre sus desempeños de comprensión con el fin de mejorarlos.

\section{Equilibrio Ácido Base}

Desde un aspecto general se puede considerar que el equilibrio químico "explica un gran número de fenómenos naturales, y desempeña un importante papel en muchos procesos industriales" (Brown, 2004). Un sistema se encuentra en equilibrio químico si se cumplen las siguientes condiciones: su estado energético es mínimo, es decir cuando $\Delta G^{\circ} \leq 0$ y cuando la velocidad de formación de productos es igual a la velocidad de la reacción contraria. 
El equilibrio ácido-base, centra su atención hacia las concentraciones de hidrogeniones $\left(\mathrm{H}^{+}\right)$hidroxilos $\left(\mathrm{OH}^{-}\right)$presentes en un sistema acuoso. Es claro que "la concentración de ion hidronio tiene dominio en la mayoría de reacciones químicas (neutralización, hidrólisis, separaciones analíticas, oxidaciones, bloqueo de grupos básicos en reacciones enzimáticas, etc.) y en la velocidad con la que proceden dichas reacciones 0 en el mecanismo por el cual ocurren" (Clavijo, 2002).

Las reacciones de neutralización, también conocidas como reacciones de transferencia protónica se soportan en el concepto ácido-base de Brønsted-Lowry, definiendo a un ácido como especie química capaz de transferir un protón a una base, la cual es otra especie química con disponibilidad, por lo menos, de un par electrónico.
Una curva de valoración fotométrica es una gráfica de absorbancia (corregida con respecto al cambio de volumen) en función de volumen del valorante (Skoog, 2008).

\section{Objetivos}

\section{General}

Diseñar e implementar una propuesta de enseñanza para el tópico generativo equilibrio ácido base, en el marco del modelo de EpC, con estudiantes de grado 11.

\section{Específicos}

- Identificar las concepciones previas de colegio Champagnat en relación con aspectos asociados al equilibrio ácido-base

- Diseñar desempeños de comprensión a través de un torbellino de ideas.

- Caracterizar los niveles de comprensión de los estudiantes después de aplicada la estrategia de enseñanza

\section{METODOLOGÍA}

\begin{tabular}{|c|c|}
\hline Información Demográfica & $\begin{array}{l}\text { Colegio: Champagnat sede Bogotá } \\
\text { Grado: } 11^{\circ} \\
\text { Tiempo duración: Agosto } 2011 \text { Julio } 2012 \\
\text { Cantidad de estudiantes: } 93\end{array}$ \\
\hline $\begin{array}{l}1^{\text {ra }} \text { Etapa } \\
\text { (Exploratoria) }\end{array}$ & $\begin{array}{l}\text { i Diseño de red de ideas para la delimitación del tópico generativo } \\
\text { ii Diseño y aplicación del instrumento de ideas previas (anexo 1) y análisis de } \\
\text { resultados. }\end{array}$ \\
\hline $\begin{array}{l}2^{\text {da }} \text { Etapa } \\
\text { (Diseño y aplicación de la } \\
\text { estrategia) }\end{array}$ & $\begin{array}{l}\text { i Diseño de desempeños y niveles de comprensión (torbellino de ideas). } \\
\text { ii Diseño del plan de cátedra. } \\
\text { 1. Cátedra por parte del profesor. } \\
\text { 1.1 Ley de Lambert-Beer. } \\
\text { 1.2 Diferencia entre absorbancia y transmitancia. } \\
\text { 1.3 Equilibrio químico homogéneo y heterogéneo. } \\
\text { 1.4 Cinética y equilibrio químico. } \\
\text { 1.5 Teorías ácido-base. } \\
\text { 1.6 Escala pH y pOH. } \\
\text { 1.7 Volumetrías y fotometrías. } \\
\text { 2. Aplicación de talleres teóricos. } \\
\text { 2.1 Lecturas y síntesis. } \\
\text { 2.2 Ejercicios de lápiz y papel. } \\
\text { 2.3 Mini-test de conocimiento. } \\
\text { 3. Diseño de taller experimental. } \\
\text { 4. Test de conocimientos finales abordados en el transcurso de la unidad } \\
\text { (anexo 2). }\end{array}$ \\
\hline $\begin{array}{l}3^{\text {ra }} \text { Etapa } \\
\text { Procesamiento y análisis de } \\
\text { resultados }\end{array}$ & $\begin{array}{l}\text { i) Sistematización de resultados obtenidos con los instrumentos aplicados } \\
\text { ii) Contrastación de los resultados obtenidos en las pruebas de entrada y salida. }\end{array}$ \\
\hline $\begin{array}{l}4^{\text {ta }} \text { Etapa } \\
\text { Evaluación de resultados }\end{array}$ & rollo de la p \\
\hline
\end{tabular}


El profesor y los estudiantes diseñan una red de ideas, con el propósito de seleccionar un tópico de interés general que oriente la ruta inicial para este proyecto y posterior diseño de la unidad.

En cuanto a la caracterización de las ideas previas (anexo 1), se identifica que, los estudiantes de grado once, tienen dificultades en la lectura de instrumentos de medición volumétrica, no interpretan la escala logarítmica, manifiestan una visión unidireccional del equilibrio químico, además de no reconocer la característica ondulatoria y corpuscular de la luz, todo lo anterior muestra la necesidad de estructurar una propuesta metodológica para el tópico generativo de equilibrio ácido-base desde la $\mathrm{EpC}$, la cual es asumida como el enfoque educativo de la Institución.

Posterior al análisis de los resultados obtenidos de la población objetivo, se diseña e implementa el plan de cátedra, descrito en la metodología de este proyecto, que orienta el trabajo hacia el logro de los desempeños de compresión que se presentan en el torbellino de ideas (tabla 2), que permite clasificar a los estudiantes en los niveles de comprensión.

En este orden de ideas, se estructuran tres niveles de compresión, que se categorizan de la siguiente manera: un puntaje de 0 a 6 clasifica a la población en novato, de más de 6 a 8 en aprendiz y de más de 8 a 10 en experto.

Uno de los principales propósitos del tópico generativo y del plan de cátedra, es que el grupo de estudiantes establezca relaciones entre las diferentes técnicas analíticas para comprender el equilibrio ácido-base, es decir, además de utilizar los métodos tradicionales, para determinar las propiedades de las reacciones de neutralización, $\mathrm{K}_{\mathrm{a}}$ - $\mathrm{K}_{\mathrm{b}}, \mathrm{pH}$ y otros parámetros asociados (Principio de Le Chatelier), es utilizar los métodos fotométricos como técnica complementaria, que promueva el desarrollo de un nivel mayor en el grado de compresión.

Una de las estrategias didácticas que promueve y potencia los desempeños de compresión, es el trabajo práctico de laboratorio, que permite evidenciar las diferencias en cuanto al grado de precisión y exactitud de los métodos tradicionales (titulaciones volumétricas), y métodos instrumentales (titulaciones potenciométricas y titulaciones fotométricas) y que permiten asociar el comportamiento de los sistemas en equilibrio dinámico, en particular lo relacionado con las reacciones de neutralización a través de modelos matemáticos (función lineal).

A continuación se muestran en la gráfica $\mathrm{N}^{\circ} 1$, a modo de ejemplo, los resultados obtenidos por los estudiantes en la titulación fotométrica del sistema ácido-base fuerte $(\mathrm{HCl} / \mathrm{KOH})$.

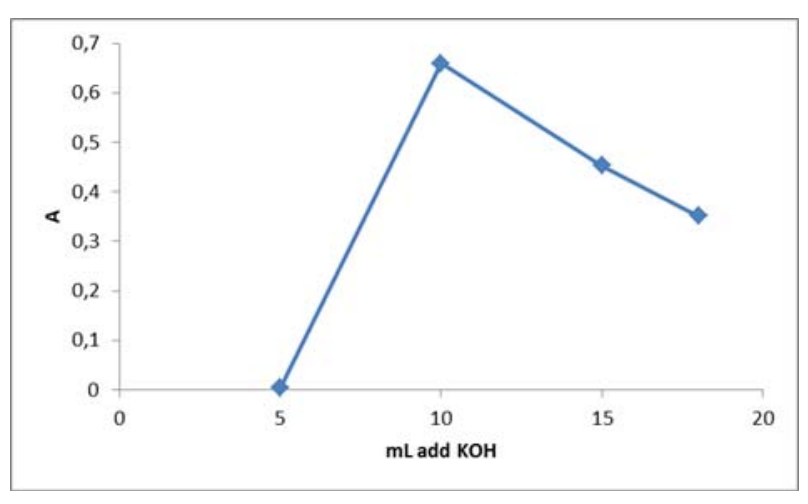

Gráfica 1. Curva de valoración fotométrica. 
Tabla 2. Torbellino de ideas

\begin{tabular}{|c|c|c|c|}
\hline \multicolumn{4}{|c|}{ Metas de comprensión abarcadoras o hilos conductores } \\
\hline & \multicolumn{3}{|c|}{ Los estudiantes comprenderán la importancia del análisis químico en equilibrios ácido base } \\
\hline $\mathrm{E}$ & \multicolumn{3}{|c|}{ Desempeños de comprensión } \\
\hline $\mathrm{U}$ & \multicolumn{3}{|c|}{ ¿Qué implicaciones y cuál es la importancia del equilibrio químico ácido-base? } \\
\hline I & Novato & Aprendiz & Experto \\
\hline $\begin{array}{l}\text { I } \\
\text { B } \\
\mathrm{R} \\
\mathrm{I} \\
\mathrm{O}\end{array}$ & $\begin{array}{l}\text { 1. Expone ideas de manera } \\
\text { argumentada en forma } \\
\text { oral y escrita. } \\
\text { 2. Desarrolla habilidades } \\
\text { de trabajo en equipo. } \\
\text { 3. Demuestra interés por } \\
\text { las temáticas trabaja- } \\
\text { das. }\end{array}$ & $\begin{array}{l}\text { 1. Expone sus habilidades } \\
\text { en el contexto de ma- } \\
\text { nera individual y grupal. } \\
\text { 2. Reconoce el significado } \\
\text { de un equilibrio químico } \\
\text { su relación con la ciné- } \\
\text { tica y los factores que } \\
\text { lo alternan (Principio de } \\
\text { Le Chatelier). } \\
\text { 3. Argumenta y diferencia } \\
\text { la definición de un áci- } \\
\text { do y una base desde } \\
\text { diversas teorías. }\end{array}$ & $\begin{array}{l}\text { 1. Reconoce la diferencia entre un } \\
\text { ácido y una base de manera ar- } \\
\text { gumentada y a través de algorit- } \\
\text { mos matemáticos que los defi- } \\
\text { nen. } \\
\text { 2. Define la constante de equilibrio } \\
\text { como una variable termodinámi- } \\
\text { ca de una reacción química. } \\
\text { 3. Relaciona situaciones de su coti- } \\
\text { dianidad y las asocia al fenó- } \\
\text { meno de un equilibrio químico } \\
\text { ácido-base. }\end{array}$ \\
\hline B & & & \\
\hline $\mathrm{E}$ & \multicolumn{3}{|c|}{$\begin{array}{l}\text { ¿Cuál es la diferencia de la titulación fotométrica con las demás } \\
\text { metodologías de análisis? }\end{array}$} \\
\hline $\begin{array}{l}E \\
S \\
P \\
C \\
T \\
R \\
O\end{array}$ & $\begin{array}{l}\text { 1. Reconoce las reacciones } \\
\text { de neutralización. } \\
\text { 2. Utiliza métodos analíti- } \\
\text { cos tradicionales para } \\
\text { determinar el pH de una } \\
\text { muestra. }\end{array}$ & $\begin{array}{l}\text { 1. Comprende el comporta- } \\
\text { miento de la luz. } \\
\text { 2. Comprende el fenómeno } \\
\text { de interacción materia- } \\
\text { energía. }\end{array}$ & $\begin{array}{l}\text { 1. Comprende y asocia el principio } \\
\text { físico-químico con el que funcio- } \\
\text { na un espectrofotómetro. } \\
\text { 2. Utiliza los métodos fotométricos } \\
\text { para determinar el pH en el punto } \\
\text { de equivalencia. }\end{array}$ \\
\hline $\begin{array}{l}\mathrm{O} \\
\mathrm{T} \\
\mathrm{O}\end{array}$ & \multicolumn{3}{|c|}{ ¿Qué implica la validez de un método analítico? } \\
\hline $\begin{array}{l}\mathrm{M} \\
\mathrm{E} \\
\mathrm{T} \\
\mathrm{R} \\
\mathrm{I} \\
\mathrm{A}\end{array}$ & $\begin{array}{l}\text { 1. Utiliza los datos estadís- } \\
\text { ticos para reportar re- } \\
\text { sultados de un labora- } \\
\text { torio. } \\
\text { 2. Maneja adecuadamente } \\
\text { instrumentos de labora- } \\
\text { torio. }\end{array}$ & $\begin{array}{l}1 \text { Hace uso de las herra- } \\
\text { mientas estadísticas y } \\
\text { las funciones que inter- } \\
\text { vienen en un análisis } \\
\text { químico. }\end{array}$ & $\begin{array}{l}\text { 1. Reconoce las herramientas esta- } \\
\text { dísticas y las funciones que inter- } \\
\text { vienen en un análisis químico } \\
\text { espectrofotométrico. } \\
\text { 2. Determina la importancia del } \\
\text { análisis estadístico en la determi- } \\
\text { nación de la concentración de } \\
\text { una muestra. }\end{array}$ \\
\hline
\end{tabular}


Aunque la evaluación del proceso es permanente, la prueba final permite indagar acerca del tópico de cinética y su relación con el equilibrio químico. Los siete puntos se estructuran de la siguiente manera:

- Interpretación y argumentación de gráficos para los puntos 1 y 5 .

- El uso de algoritmos matemáticos que definen un constante de equilibrio, tanto para sistemas homogéneos como para heterogéneos, puntos 2,3 y 4 .

- Preguntas asociadas al principio de Le Chatelier aplicados a cualquier equilibrio puntos 6 y 7 (Anexo 2)

En la gráfica 2 se observa que, después de desarrollar el plan de cátedra y aplicar la prueba final, el grupo de estudiantes presenta tres grados de desempeño para el tópico generativo equilibrio ácido-base, se muestra que el $24,10 \%$ de la población se ubica en nivel novato, $24,10 \%$ se ubican en el nivel de experto y el $51,81 \%$ se ubica en un nivel de aprendiz.



Gráfica 2. Niveles de comprensión, en porcentaje, alcanzados por el grupo de estudiantes.
Los resultados de la prueba final muestran que los estudiantes, objeto de este estudio, se encuentran en nivel de aprendiz (Tabla 3 ) en relación con los desempeños de comprensión orientados a través de la estrategia de enseñanza.

\begin{tabular}{|cc|}
\hline Media & 7 \\
\hline Mediana & 7,1 \\
\hline Moda & 6,36 \\
\hline Desviación Media & 1,3 \\
\hline Valor Mínimo & 3,2 \\
\hline Valor Máximo & 9,48 \\
\hline
\end{tabular}

Tabla 3. Parámetros estadísticos del test final de conocimientos de acuerdo a la calificación.

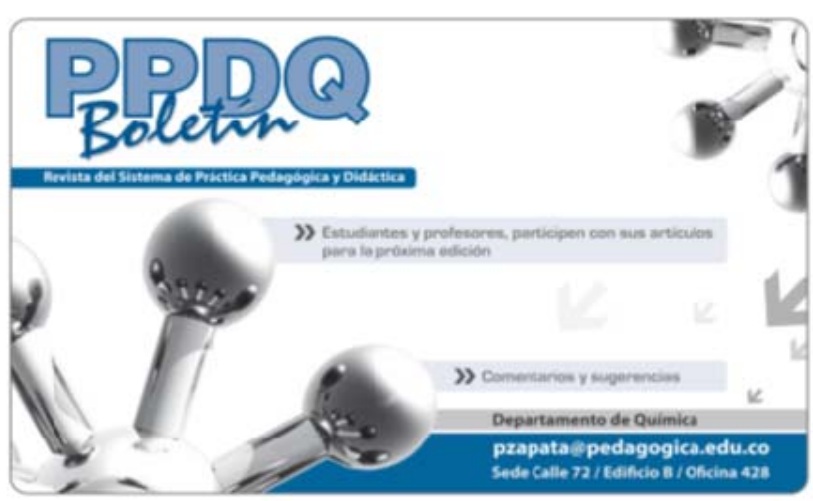


La utilización de actividades, de tipo conceptual y experimental, propician desempeños de compresión en relación con el tópico generativo de equilibrio ácidobase, dado que se encuentra que el nivel de compresión del grupo de estudiantes es aprendiz, después de la implementación de la estrategia..

El torbellino de ideas, como herramienta fundamental de la $\mathrm{EpC}$, favorece la orientación del diseño metodológico articulado con los desempeños de compresión.
El diseño y la implementación de la propuesta basada en la EpC, se consolida como una aproximación curricular para la transformación de las prácticas educativas y aporta en el proceso de formación en química de los estudiantes del Colegio Champagnat, puesto que estos comprenden el significado del equilibrio químico, su relación con la cinética y los factores que lo alternan (Principio de Le Chatelier); comprenden el comportamiento de la luz y el fenómeno de interacción materiaenergía, esto se evidencia al contrastar con los resultados obtenidos con el test de ideas previas.

\section{BIBLIOGRAFÍA}

Brown (2004). Química la ciencia central. Pearson. London.

Clavijo, A (2002). Fundamentos de química analítica. Universidad Nacional. Bogotá.

Martínez, J (2007). Enseñanza para la compresión una aplicación en el aula. Universidad Pedagógica Nacional. Bogotá.
Murillo, J (2006).La formación de docentes: una clave para la mejora educativa. Chile.

Colegio Champagnat (2002). Proyecto Educativo Institucional Sede Bogotá.

Skoog, W.(2008). Principios de Análisis instrumental. México: Ciengage.

Stone, W. (2005). Enseñanza para la comprensión: Vinculación entre la investigación y la práctica. Buenos Aires, Argentina: Paidós. 


\section{Anexo 1}

\section{Prueba de Ideas Previas-Etapa Exploratoria}

El siguiente cuestionario debe ser resuelto de manera individual y sincera. Gracias por su colaboración.

1.- De acuerdo con las propiedades físicas y químicas de las sustancias, como densidad, polaridad, viscosidad además del comportamiento de las sustancias puras y en disolución, ¿en cuál de los siguientes tubos se puede realizar una lectura apropiada teniendo en cuenta que se está teniendo un vista horizontal la altura de los ojos con la línea trazada a través de cada uno de los tubos. Marque con una $\mathrm{X}$ la situación que mejor represente la lectura correcta

\begin{tabular}{|c|c|}
\hline $\begin{array}{c}\text { Volumen registrado } \\
1 \mathrm{~mL}\end{array}$ & $\begin{array}{c}\text { Volumen registrado } \\
\mathbf{1 , 1} \mathrm{mL}\end{array}$ \\
\hline \multirow{2}{*}{$\mathrm{A}$} & \\
\hline
\end{tabular}

2.- La precisión y la exactitud son aspectos fundamentales en la lectura científica, por ejemplo para preparar unas disolución se requiere exactamente un concentración en masa del $1 \%$ se diluye $1 \mathrm{~g}$ de soluto en $100 \mathrm{~g}$ de solución (de una especie ácida), suponiendo que esta es la dosis necesaria para neutralizar una concentración de $1 \%$ en masa de una especie básica. De acuerdo con la información anterior y sus conocimientos, relacione las gráficas y ubique la letra correspondiente en cada uno de los recuadros pequeños para cada una de las siguientes opciones.

\section{Gráfico de precisión y exactitud}

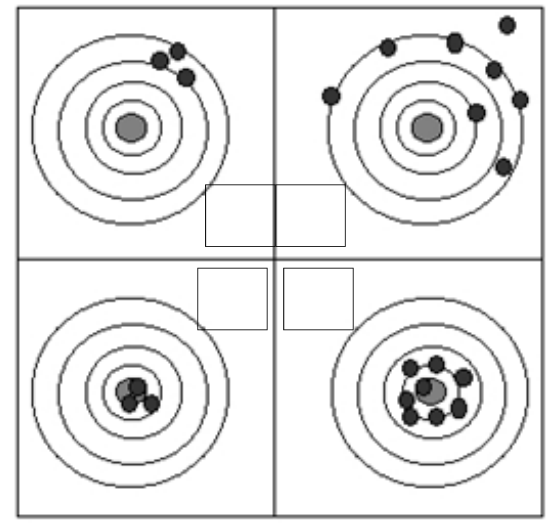

-Alta exactitud y baja precisión

- Baja exactitud y alta precisión

-Alta exactitud y alta precisión

- Baja exactitud y baja precisión

3.- El pH se define como logaritmo negativo de la concentración de hidrogeniones, que se calcula con la siguiente igualdad $\mathbf{P}^{\mathrm{H}}=-\log \mathbf{H}^{+}$

¿Cual es el resultado de pH para la concentración de iones hidrogenión de 3,0 X10 $0^{-3} \mathrm{M}$ ?

El pH es bajo por lo tanto la dilución es acida.

El pH se bajo por lo tanto la dilución no es acida.

2,52

2,523 
4.- Las sustancias iónicas en disolución con agua se disocian en sus iones constitutivos, dicho proceso se evidencia por el transporte efectivo en diversos tipos de membrana en la célula, con base en la anterior información y su conocimiento, indique en el recuadro, el sentido en que se desarrolla la dilución, en agua, de sal de cocina $(\mathrm{NaCl})$

$$
\mathrm{NaCl}_{(\mathrm{s})}+\mathrm{H}_{2} \mathrm{O}_{(1)}
$$

A) $\longrightarrow$

B)

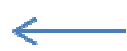

$\mathrm{Na}^{+}{ }_{(\mathrm{ac})}+\mathrm{Cl}^{-}$

C)

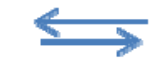

(ac)

D)

Justifique su respuesta

5.- De acuerdo con las propiedades físico-químicas que caracterizan la fase en la que se encuentran cada una de las especies químicas de los reactantes y productos de una reacción, el cual es definido por la constante de equilibrio, clasifique las siguientes reacciones químicas como homogéneas $(O)$ y heterogéneas $(E)$ en la columna de la derecha. Justifique su respuesta

\begin{tabular}{|l|l|}
\hline $2 \mathrm{SO}_{2(\mathrm{~g})}+\mathrm{O}_{2(\mathrm{~g})} \longleftrightarrow \mathbf{2 ~ S O}_{3(\mathrm{~g})}$ & \\
\hline $\mathrm{AgCl}_{(\mathrm{s})} \longleftrightarrow \mathrm{Ag}_{(\mathrm{ac})}^{+}+\mathrm{Cl}_{(\mathrm{ac})}^{-}$ & \\
\hline $2 \mathrm{H}_{2(\mathrm{~g})}+\mathrm{O}_{2(\mathrm{~g})} \longleftrightarrow \mathrm{2H}_{2} \mathrm{O}_{(\mathrm{l})}$ & \\
\hline $\mathrm{CH}_{3} \mathrm{COOH}_{(\mathrm{ac})} \longleftrightarrow \mathrm{H}_{(\mathrm{ac})}^{+}+\mathrm{CH}_{3} \mathrm{COO}_{(\mathrm{ac})}^{-}$ & \\
\hline
\end{tabular}

6.- Las especies químicas, dada su naturaleza espectrofotométrica, absorben determinada longitud de onda lo que permite su clasificación, teniendo en cuenta que el ojo humano percibe un pequeño fragmento de espectros de emisión debido a la descomposición de la luz blanca. En la casilla llamada "Tipo de radiación" ubicada en el gráfico siguiente, escriba cada uno de los tipos de radiación que se encuentran como opciones en la tabla, hacia la izquierda.

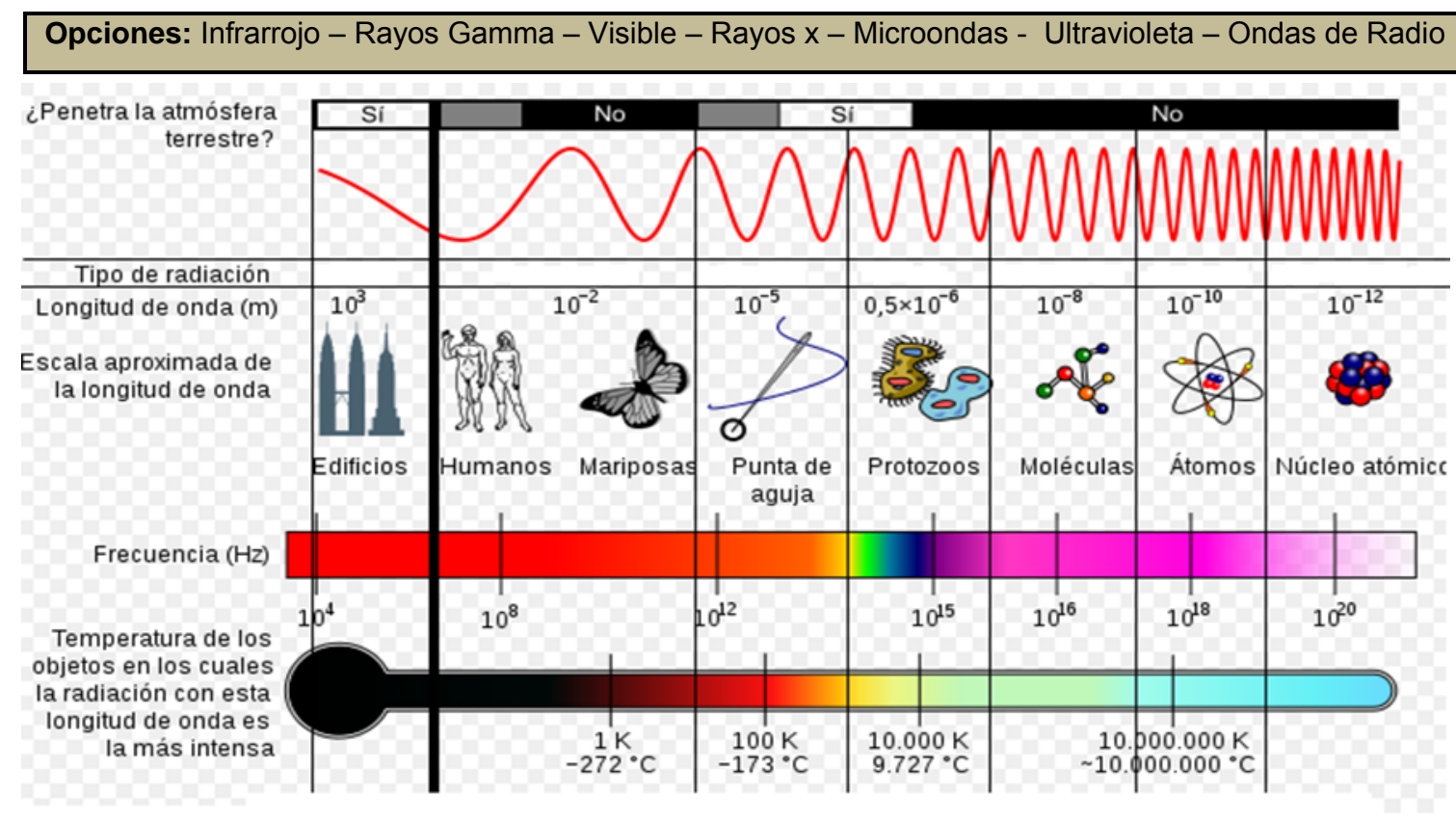

EM_Spectrum_Properties_es.svg (SVG file, nominally $700 \times 400$ pixels, file size: $185 \mathrm{~KB}$ ) 


\section{Prueba Final}

1.Con respecto a la gráfica, qué puede inferir usted con respecto a: ¿las concentraciones de los reactivos y de los productos?, ¿con respecto a la velocidad de la reacción?. ¿Cuál es la expresión para calcular la velocidad de la reacción en el equilibrio?

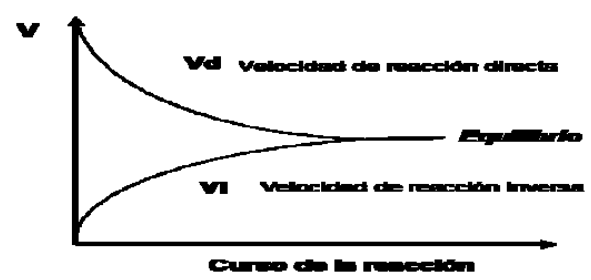

2.Formule la expresión para calcular la velocidad de la reacción en términos de los reactivos y de los productos. Explique:

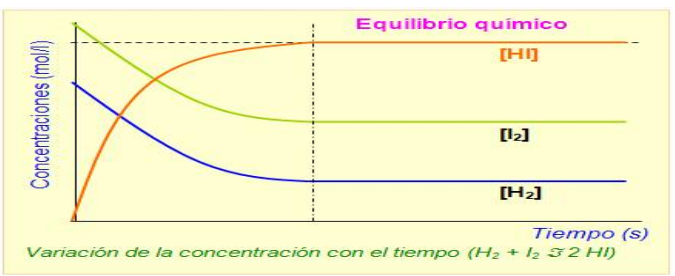

3. Si consideramos el equilibrio a $1000^{\circ} \mathrm{C}$ :

$$
\begin{gathered}
2 \mathrm{SO}_{2(\mathrm{~g})}+\mathrm{O}_{2(\mathrm{~g})} \leftrightarrow 2 \mathrm{SO}_{3(\mathrm{~g})} \\
0,34 \mathrm{M} \quad 0,17 \mathrm{M} \quad 0,06 \mathrm{M}
\end{gathered}
$$

La expresión del equilibrio $(\mathrm{Ke})$ ¿a qué es igual? y según el análisis realizado, ¿el equilibrio es homogéneo o es heterogéneo? Explique:

4.En el proceso de formación del amoniaco, realizado a $500{ }^{\circ} \mathrm{C}$ en un recipiente de 10 litros, se han encontrado en el equilibrio 6 moles de $\mathrm{N}_{2}, 4$ moles de $\mathrm{H}_{2}$ y 1,12 moles de $\mathrm{NH}_{3}$. ¿Cuál será el rendimiento de esa reacción a $500{ }^{\circ} \mathrm{C}$ ? Explique los valores de $(K)$ para el desplazamiento de la reacción.

$$
\mathrm{N}_{2(\mathrm{~g})}+3 \mathrm{H}_{2(\mathrm{~g})} \leftrightarrow 2 \mathrm{NH}_{3(\mathrm{~g})}
$$

5. Sobre la gráfica de cinética química, represente los elementos requeridos para explicar la variación de la energía en reacciones exotérmicas y endotérmicas, y, la incidencia de catalizadores e inhibidores. Explique:



6.

$$
\mathrm{X}_{2}+\mathrm{Y}_{2} \leftrightarrow 2 \mathrm{XY}
$$

Si reacciona $1 \mathrm{~mol}$ de $X_{2}$ con $1 \mathrm{~mol}$ de $Y_{2}$ hasta llegar al equilibrio, la gráfica que describe correctamente este proceso en el tiempo (t) es:

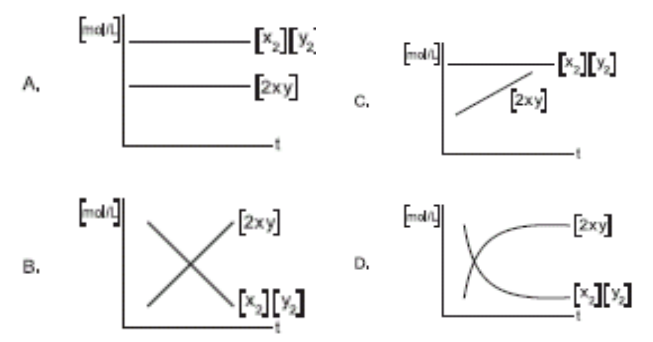

7. $\mathrm{X}$ y $\mathrm{W}$ reaccionan de acuerdo con la siguiente ecuación:

$$
X_{(s c)}+2 W_{(s c)} \rightarrow Z \downarrow+T_{(s c)}
$$

La gráfica que representa adecuadamente la reacción entre X y W es:
A.

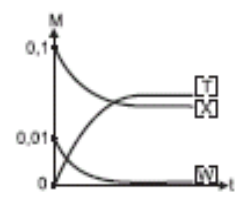

в.



c.

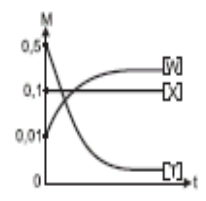

D.

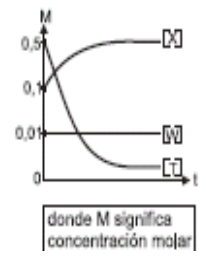

